\title{
IQ imbalance compensation for OFDM based wireless LANs
}

\author{
Jiho Kim $^{1 \mathrm{a})}$, Yunho Jung ${ }^{2}$, Byungjik Son ${ }^{3}$, and Jaeseok Kim ${ }^{1}$ \\ ${ }^{1}$ Electrical and Electronic Engineering, Yonsei University, \\ 134 Shinchon-dong, Seodaemun-gu, Seoul 120-749, Republic of Korea \\ ${ }^{2}$ Institute of TMS Information Technology, Yonsei University, \\ 134 Shinchon-dong, Seodaemun-gu, Seoul 120-749, Republic of Korea \\ ${ }^{3}$ Samsung Electronics Co., Ltd., Dong Suwon P.O.BOX 105, 416 Maetan-3dong, \\ Paldal-gu, Suwon-city, Gyeonggi-do 442-600, Republic of Korea \\ a)jhkim0518@yonsei.ac.kr
}

Abstract: Zero-IF architecture has become popular for WLAN receivers due to its simplicity. However, zero-IF receivers suffer from the effect of IQ imbalance and carrier frequency offset which may have huge impacts on their performance. Several joint compensation schemes for these influences have been researched, based on the correlation between adjacent channel frequency responses. However, by the limitation assuming the perfect symbol synchronization, these schemes show performance degradation when a symbol timing error exists. In this paper, we analyze the effect of symbol timing errors and propose a robust and efficient compensation scheme. Simulation results show the proposed algorithm achieves an SNR gain of $7.3 \mathrm{~dB}$ over the conventional algorithm when a symbol timing error occurs.

Keywords: IQ imbalance, zero-IF, OFDM, WLAN

Classification: Science and engineering for electronics

\section{References}

[1] C.-L. Liu, "Impacts of I/Q Imbalance on QPSK-OFDM-QAM Detection," IEEE Trans. Consum. Electron., vol. 44, no. 3, pp. 984-989, Aug. 1998.

[2] T. Wakutsu and M. Serizawa, "A Carrier Frequency Offset and Timing Offset Detection Scheme for OFDM Systems Utilizing Pilot SubCarriers," IEICE Trans. Commun., vol. E83-B, no. 8, pp. 1854-1863, Aug. 2000.

[3] J. Tubbax, A. Fort, L. Van der Perre, S. Donnay, M. Engels, M. Moonen, and H. De Man, "Joint compensation of IQ imbalance and frequency offset in OFDM systems," GLOBECOM '03, vol. 4, pp. 2365-2369, Dec. 2003.

[4] J. Lee, H. Lou, and D. Toumpakaris, "Maximum likelihood estimation of time and frequency offset for OFDM systems," IEEE Electron. Lett., vol. 40, pp. 1428-1429, Oct. 2004.

[5] IEEE 802.11a, Wireless LAN MAC and PHY Specifications, 1999. 


\section{Introduction}

Orthogonal frequency division multiplexing (OFDM) is sensitive to nonidealities in the receiver front-end, which leads either to stringent analog front-end specifications or to severe performance degradation. In particular, IQ imbalance and carrier frequency offset (CFO) have been identified as key front-end effects in OFDM systems, causing inter-carrier interference (ICI) $[1,2]$. In order to compensate jointly for the IQ imbalance and CFO, the IQ-FD algorithm [3], with its assumption of perfect timing synchronization, has been proposed for OFDM-based wireless LAN (WLAN) systems. Although the IQ-FD algorithm can improve system performance dramatically by compensating for IQ imbalance in the presence of CFO, a symbol timing error (STE) should be considered because it is not practical to find the exact starting point of an OFDM symbol, due to the statistical properties of the multipath fading channel [4]. Since the IQ-FD algorithm shows serious performance degradation in the presence of a STE, it should be modified to be robust with respect to STEs. In this paper, we propose an efficient IQ imbalance compensation algorithm which considers the effects of STEs. We derive a modified criterion to remove the effect of the STE efficiently, showing almost the same bit error rate (BER) performance as the ideal case where no IQ imbalance, CFO and STE exist.

\section{The IQ-FD algorithm}

The received signal $r_{n f o-i q}$ with IQ imbalance in the presence of CFO is given by $[3]$

$$
r_{n f o-i q}=\alpha \cdot r \cdot e^{j 2 \pi f_{n f o} n / N}+\beta \cdot r^{*} \cdot e^{-j 2 \pi f_{n f o} n / N},
$$

where $\alpha=\cos \theta+j \varepsilon \sin \theta$ and $\beta=\varepsilon \cos \theta-j \sin \theta$, respectively. Through the rest of the paper, IQ parameters refer to $\alpha$ and $\beta$. $\varepsilon$ and $\theta$ are the gain and phase imbalance, respectively. $f_{n f o}$ denotes the normalized CFO to the subcarrier spacing of the regular OFDM symbols with $N$ subcarriers, and $(\cdot)^{*}$ stands for the complex conjugate. Assuming that the normalized CFO can be perfectly estimated by time-domain processing, the CFO compensated signals, $r_{1}$ and $r_{2}$, with the estimated $-f_{n f o}$ and $+f_{n f o}$ are given by

$$
\begin{aligned}
& r_{1}=r_{n f o-i q} \cdot e^{-j 2 \pi f_{n f o} n / N}=\alpha \cdot r+\beta \cdot r^{*} \cdot e^{-2 j 2 \pi f_{n f o} n / N}, \\
& r_{2}=r_{n f o-i q} \cdot e^{j 2 \pi f_{n f_{o} n / N}}=\alpha \cdot r \cdot e^{2 j 2 \pi f_{n f o} n / N}+\beta \cdot r^{*} .
\end{aligned}
$$

If we can perfectly estimate the IQ parameters, $\alpha$ and $\beta$, the IQ imbalance compensated signal can be derived from Eqs. (2) and (3)

$$
\hat{r}=\frac{\alpha^{*} \cdot r_{1}-\beta \cdot r_{2}^{*}}{|\alpha|^{2}-|\beta|^{2}}
$$

In order to estimate the IQ parameters, channel frequency response (CFR) is introduced. The CFR, $c_{k}$, for the $k$ th subcarrier can be achieved from least square (LS) estimation of the received long training symbol (LTS) [5] and is given by

$$
c_{k}=f f t\left[r_{l t s}\right]_{k} \cdot l t s_{k}
$$


where $r_{l t s}$ is the received LTS, $l t s_{k}$ is the known LTS for the $k$ th subcarrier, and $f f t[\cdot]_{k}$ refers to the fast Fourier transform (FFT). To compensate for the effect of the CFO, we use the derivatives, Eqs. (2) and (3)

$$
\begin{aligned}
c_{1, k} & =f f t\left[r_{l t s, 1}\right]_{k} \cdot l t s_{k} \\
& =f f t\left[\alpha \cdot r_{l t s}+\beta \cdot r_{l t s}^{*} \cdot e^{-2 j 2 \pi f_{n f o} n / N}\right]_{k} \cdot l t s_{k} \\
& =\alpha \cdot c_{k}+l t s_{k} \cdot \beta \cdot f f t\left[r_{l t s}^{*} \cdot e^{-2 j 2 \pi f_{n f o} n / N}\right]_{k}, \\
c_{2, k} & =f f t\left[r_{l t s, 2}^{*}\right]_{k} \cdot l t s_{k} \\
& =f f t\left[\alpha^{*} \cdot r_{l t s}^{*} \cdot e^{-2 j 2 \pi f_{n f o} n / N}+\beta^{*} \cdot r_{l t s}\right]_{k} \cdot l t s_{k} \\
& =l t s_{k} \cdot \alpha^{*} \cdot f f t\left[r_{l t s}^{*} \cdot e^{-2 j 2 \pi f_{n f o} n / N}\right]_{k}+\beta^{*} \cdot c_{k},
\end{aligned}
$$

where $r_{l t s, 1}$ and $r_{l t s, 2}$ are the CFO compensated signals of $\mathrm{r}_{l t s}$. From Eqs. (6) and (7), the IQ imbalance compensated CFR, $\hat{c}_{k}$, can be derived as

$$
\hat{c}_{k}=\frac{\alpha^{*} \cdot c_{1, k}-\beta \cdot c_{2, k}}{|\alpha|^{2}-|\beta|^{2}}
$$

The estimation of the IQ parameters is based on the assumption that the compensated CFR should have a smooth channel characteristic. In other words, the optimal $\alpha$ and $\beta$ should minimize the difference between the adjacent CFRs. As a result, the IQ-FD algorithm criterion to find the IQ parameters is given by

$$
M S E=\sum_{k=1}^{N-1}\left|\hat{c}_{k+1}-\hat{c}_{k}\right|,
$$

and the IQ parameters which minimize the criterion are given by

$$
\begin{gathered}
\beta=\frac{\sum_{k=1}^{N}\left(c_{1, k+1}-c_{1, k}\right)\left(c_{2, k+1}^{*}-c_{2, k}^{*}\right)}{\sum_{k=1}^{N}\left|c_{2, k+1}-c_{2, k}\right|^{2}}, \\
\alpha=\sqrt{1-\operatorname{Im}^{2}\{\beta\}}-j \frac{\operatorname{Re}\{\beta\} \operatorname{Im}\{\beta\}}{\sqrt{1-\operatorname{Im}^{2}\{\beta\}}},
\end{gathered}
$$

where $\operatorname{Re}\{\cdot\}$ and $\operatorname{Im}\{\cdot\}$ denote the real and imaginary parts, respectively.

Although the IQ-FD algorithm can improve performance by correcting the received signal and CFR from Eq. (4) and Eq. (8), it suffers from the limitation that it assumes perfect symbol synchronization. As stated, since it is very hard to find the exact starting point of an OFDM symbol due to the statistical properties of the multipath fading channel, and STE destroys the assumption of the smooth channel characteristic, STE should be considered with CFO to compensate for IQ imbalance. Fig. 1 depicts the real term of CFR distorted by the effect of the STE. Since the distorted CFR does not have a smooth characteristic, the criterion, in Eq. (9), is inadequate in the presence of the STE. Therefore, a new criterion to find the IQ parameters is needed. 


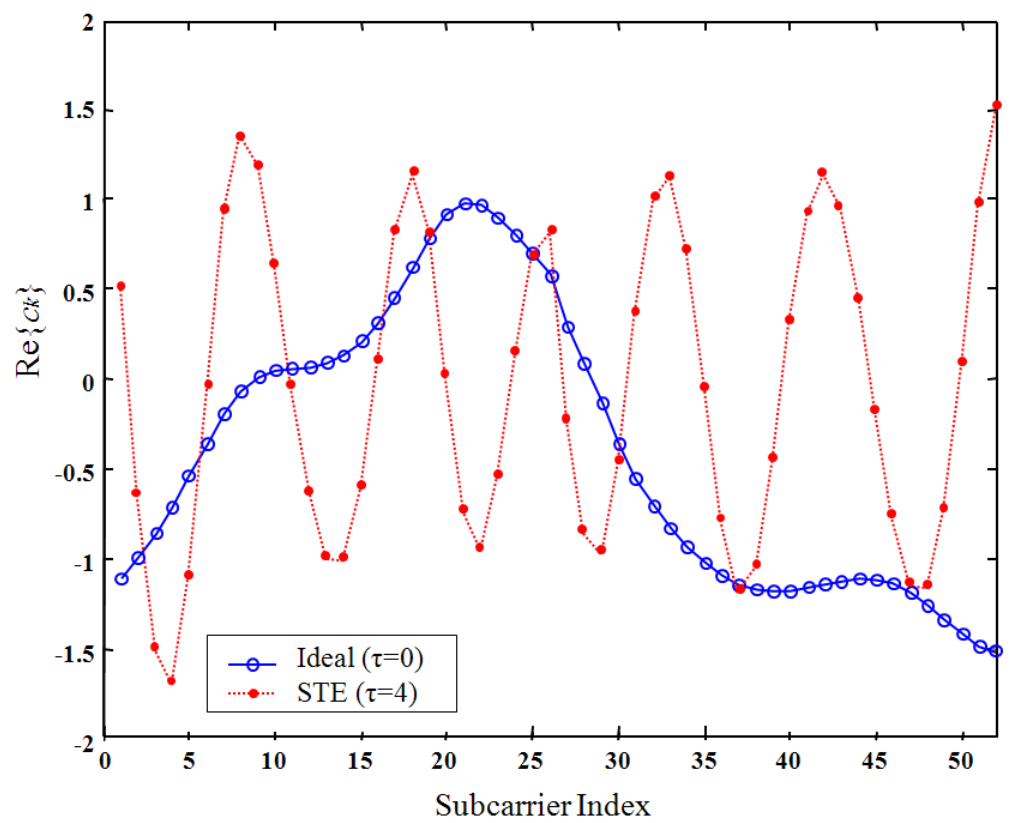

Fig. 1. Channel frequency responses distorted by the effect of the STE.

\section{Proposed IQ imbalance compensation algorithm}

The received signal $r_{n f o-i q-}$ with the effect of the IQ imbalance in the presence of CFO and STE is derived from Eq. (1)

$$
r_{n f o-i q-\tau}=\alpha \cdot r(n-\tau) \cdot e^{j 2 \pi f_{n f o}(n-\tau) / N}+\beta \cdot r^{*}(n-\tau) \cdot e^{-j 2 \pi f_{n f o}(n-\tau) / N},
$$

where $\tau$ is the STE. Similar to Eq. (2) and Eq. (3), the CFO compensated signals are given by

$$
\begin{aligned}
r_{1} & =r_{n f o-i q-\tau} \cdot e^{-j 2 \pi f_{n f o} n / N} \\
& =\alpha \cdot r(n-\tau) \cdot e^{-j 2 \pi f_{n f o} \tau / N}+\beta \cdot r^{*}(n-\tau) \cdot e^{-j 2 \pi f_{n f o}(2 n-\tau) / N}, \\
r_{2} & =r_{n f o-i q-\tau} \cdot e^{j 2 \pi f_{n f o} n / N} \\
& =\alpha \cdot r(n-\tau) \cdot e^{j 2 \pi f_{n f o}(2 n-\tau) / N}+\beta \cdot r^{*}(n-\tau) \cdot e^{j 2 \pi f_{n f o} \tau / N},
\end{aligned}
$$

and the IQ imbalance compensated signal can also be derived as

$$
\hat{r}_{\tau}=\hat{r}(n-\tau)=\frac{\alpha^{*} \cdot r_{1}-\beta \cdot r_{2}^{*}}{|\alpha|^{2}-|\beta|^{2}} .
$$

In order to estimate the IQ parameters, we take the same approaches as Eqs. (5)-(8). The CFO compensated CFRs are given by

$$
\begin{aligned}
c_{1, k} & =f f t\left[r_{l t s, 1}\right]_{k} \cdot l t s_{k} \\
& =f f t\left[\alpha \cdot r_{l t s}(n-\tau) \cdot e^{-j 2 \pi f_{n f o} \tau / N}+\beta \cdot r_{l t s}^{*}(n-\tau) e^{-j 2 \pi f_{n f o}(2 n-\tau) / N}\right]_{k} \cdot l t s_{k} \\
& =\alpha \cdot c_{\tau, k}+l t s_{k} \cdot \beta \cdot f f t\left[r_{l t s}^{*}(n-\tau) e^{-2 j 2 \pi f_{n f o} n / N}\right]_{k} \cdot e^{j 2 \pi f_{n f o} \tau / N}, \\
c_{2, k} & =f f t\left[r_{l t s, 2}^{*}\right]_{k} \cdot l t s_{k} \\
& =f f t\left[\alpha^{*} \cdot r_{l t s}^{*}(n-\tau) \cdot e^{-j 2 \pi f_{n f o}(2 n-\tau) / N}+\beta^{*} \cdot r_{l t s}(n-\tau) \cdot e^{-j 2 \pi f_{n f o} \tau / N}\right]_{k} \cdot l t s_{k} \\
& =l t s_{k} \cdot \alpha^{*} \cdot f f t\left[r_{l t s}^{*}(n-\tau) e^{-2 j 2 \pi f_{n f o} n / N}\right]_{k} \cdot e^{j 2 \pi f_{n f o} \tau / N}+\beta^{*} \cdot c_{\tau, k}, \quad(17)
\end{aligned}
$$


where $c_{\tau, k}$ is the CFR in the presence of the STE and is given by

$$
\begin{aligned}
c_{\tau, k} & =f f t\left[r_{l t s}(n-\tau) \cdot e^{-j 2 \pi f_{n f o} \tau / N}\right]_{k} \cdot l t s_{k} \\
& =f f t\left[r_{l t s}\right]_{k} \cdot e^{-j 2 \pi k \tau / N} \cdot e^{-j 2 \pi f_{n f o} \tau / N} \cdot l t s_{k} \\
& =c_{k} \cdot e^{-j 2 \pi\left(k+f_{n f o}\right) \tau / N},
\end{aligned}
$$

and the IQ imbalance compensated CFR, $\hat{c}_{\tau, k}$, can be given by Eq. (8).

As shown in Eq. (18), since the CFR in the presence of the STE suffers from phase rotation proportional to the subcarrier index, the criterion of the IQ-FD algorithm, Eq. (9), cannot give optimal IQ parameters. In order to derive a new criterion to estimate the IQ parameters in the presence of the STE, we use the following property:

$$
\measuredangle \hat{c}_{\tau, l}=\measuredangle \hat{c}_{\tau, N-l}^{*} \quad(l=1, \ldots, N / 2-1),
$$

where $\measuredangle(\cdot)$ denotes the phase term. From that property, we define a new parameter, $\lambda_{l}$, to eliminate the phase rotation caused by the STE as follows:

$$
\begin{aligned}
\lambda_{l} & =\hat{c}_{\tau, l} \cdot \hat{c}_{\tau, N-l} \\
& =\hat{c}_{l} \cdot e^{-j 2 \pi\left(l+f_{\left.n f_{o}\right) \tau / N}\right.} \cdot \hat{c}_{N-l} \cdot e^{-j 2 \pi\left(N-l+f_{n f o}\right) \tau / N} . \\
& =\hat{c}_{l} \cdot \hat{c}_{N-l} \cdot e^{-2 j 2 \pi f_{n f_{o}} \tau / N}
\end{aligned}
$$

Eq. (20) shows that $\lambda_{l}$ has constant phase rotation independent of the subcarrier index, $l$, and therefore $\lambda_{l}$ has a smooth channel characteristic. Fig. 2 shows the real term of $\lambda_{l}$ with $\tau=4$.

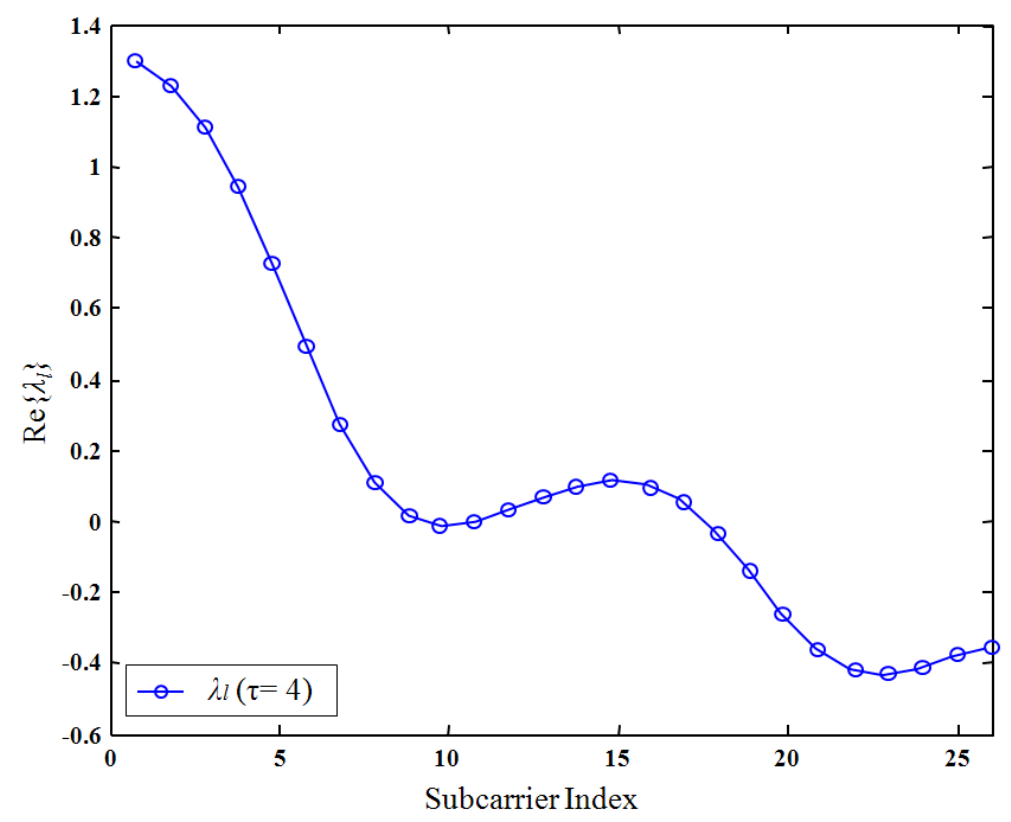

Fig. 2. The parameter $\lambda_{l}$ with $\tau=4$.

Since $\lambda_{l}$ shows a smooth characteristic similar to the CFR without STE, we can find the IQ parameters which minimize the following new criterion

$$
M S E=\sum_{l=1}^{N / 2-1}\left|\lambda_{l+1}-\lambda_{l}\right|^{2} .
$$


Consequently, we can derive $\beta$ to minimize Eq. (21)

$$
\beta=\frac{\sum_{l=1}^{N / 2-1}\left(c_{1, l+1} c_{1, N-l}-c_{1, l} c_{1, N-l+1}\right)}{\left.\sum_{l=1}^{N / 2-1} \mid c_{1, N-l} c_{2, l+1}-c_{1, N-l+1} c_{2, l}+c_{1, l+1} c_{2, N-l}-c_{2, l+1}-c_{1, N-l+1} c_{2, N-l+1}\right)^{*}+c_{1, l+1} c_{2, N-l}-\left.c_{1, l} c_{2, N-l}\right|^{2}},
$$

and by using the estimated $\beta, \alpha$ can be obtained as shown in Eq. (11). As stated, once $\alpha$ and $\beta$ are calculated, the OFDM data symbol and the CFR are compensated for the IQ imbalance by Eq. (4) and Eq. (8).

\section{Simulation Results}

In order to analyze the influence of the STE, we evaluate the BER performance for $54 \mathrm{Mb} / \mathrm{s}$ transmission mode (64 QAM, coding rate $=3 / 4$ in [5]) in a 12-tap exponentially decaying Rayleigh fading channel with $\varepsilon=0.1, \theta=10^{\circ}$, and $\mathrm{CFO}=200 \mathrm{KHz}$. Fig. 3 shows the performance of the IQ-FD and proposed algorithms in the presence of the STE. At BER of $10^{-3}$, the proposed algorithm achieves a SNR gain of $7.3 \mathrm{~dB}$ over the IQ-FD algorithm for the case of $\tau=4$. As shown in Fig. 3, with the proposed algorithm, the IQ imbalance can be totally eliminated even in the presence of CFO and STE.

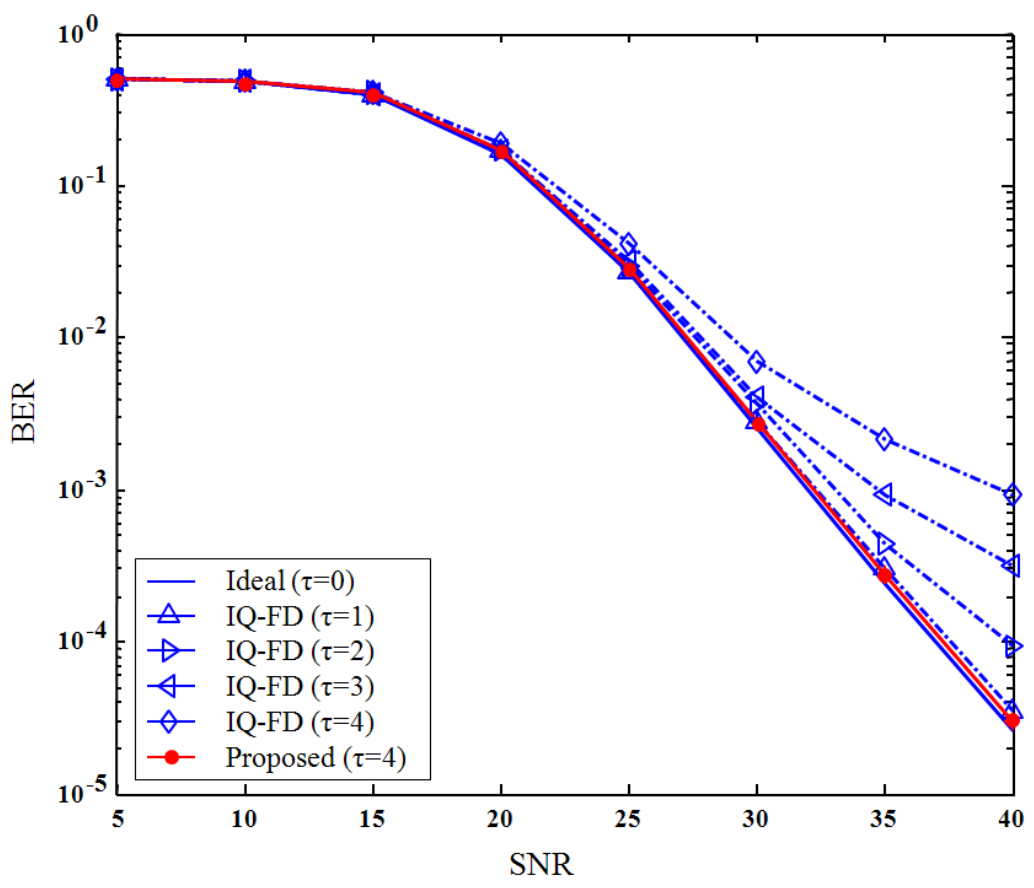

Fig. 3. BER performance of the IQ-FD and proposed algorithms for various symbol timing errors. 


\section{Conclusion}

We proposed an efficient compensation algorithm for the IQ imbalance in OFDM-based WLAN systems. To eliminating the effects of STE, we proposed a new criterion to find the IQ parameters. The proposed algorithm shows better performance than the IQ-FD algorithm. With the proposed algorithm, we can reduce the performance degradation caused by the IQ imbalance to $0.2 \mathrm{~dB}$ even in the presence of CFO and STE.

\section{Acknowledgments}

This research was supported by the MIC (Ministry of Information and Communication), Korea, under the ITRC (Information Technology Research Center) support program supervised by the IITA (Institute of Information Technology Assessment) and Yonsei University Institute of TMS Information Technology, a Brain Korea 21 program, Korea. 